\title{
Les voix dans un entretien de recherche : quelles marques et quelles fonctions interactionnelles et sociales?
}

\author{
Marie Carcassonne \\ Université Paris-Dauphine \& Institut de Recherche Interdisciplinaire en Sciences Sociales (IRISSO), UMR 7170 \\ marie.carcassonne@dauphine.fr
}

\section{Introduction}

La perspective dialogique de Bakhtine (Bakhtine-Volochinov (1977 [1929]) $\left.)^{1}\right)$ peut se définir à grands traits comme un travail interprétatif consistant à mettre en rapport les voix traversant un discours donné avec d'autres voix, qu'elles soient présentes dans ce même discours (dialogisme "interne ») ou dans d'autres discours (dialogisme «externe »). Elle renvoie à un phénomène de circulation des discours généré par leur inscription nécessaire dans un contexte social et idéologique. Cette relation d'un discours à d'autres discours peut se manifester sous forme d'échos (résonance, harmoniques) ou de voix introduisant de l'autre dans l'un. Ces échos et ces voix « retentissent » (François, 2009) sur le récepteur en faisant signe vers d'autres discours ; ils manifestent en cela une forme d'altérité (plus ou mois marquée et délimitée selon les discours). Cette hétérogénéité des voix et des sources a été caractérisée comme « constitutive » (Authier-Revuz, 1982) ${ }^{2}$ de tout discours. Elle peut être mise en lien avec le phénomène de « double résidu » pointé par Eco (1992 : 30) pour qui tout discours « narcotise » des significations qui ne sont pas actualisées, tout en les laissant à l'horizon : tout discours manifeste ainsi quelque chose " qu'on peut dire aussi » (le résidu $)^{3}$ et c'est en cela qu'il permet une réponse.

L'orchestration polyphonique des voix au sens bakhtinien ${ }^{4}$ du terme - c'est-à-dire au sens où aucune des voix ne domine l'autre dans un discours - sera questionnée à partir d'un entretien de recherche en psychopathologie ; cet entretien a lieu entre un médecin, psychanalyste et chercheur, et une jeune femme d'une trentaine d'année (F.) qui a été anorexique de longues années et a eu l'occasion, au cours de plusieurs psychothérapies, de réfléchir sur son histoire et notamment sur l'origine de sa maladie. Cet entretien est traversé de bout en bout par une tension entre une voix relevant d'un discours médical et d'autres types de voix moins spécialisées pour expliquer l'apparition des troubles du comportement alimentaire. Il est caractéristique d'une attitude décrite par Clavreul comme étant celle de tout un chacun vis-à-vis de la sphère du discours médical, lequel nous mettrait dans une position subjective divisée : chacun ne demanderait qu'à s'y soumettre, sans toutefois accepter l'idée (qu'il affirmerait pour pouvoir se perpétuer) selon laquelle "le médecin (ou le chef) sait mieux que toi ce qui convient pour ton bien » (1978: 11).

Nous mobiliserons centralement les notions de mouvements discursifs et interprétatifs et de places discursives et sociales, dans une perspective dialogique, interactionnelle et énonciative (KerbratOrecchioni, 1980, 1989, 1990 ; Vion, 1999, 2001) ou plus fortement ethnométhodologique (Mondada, 1989, 2001), lesquelles sont dérivées de l'analyse conversationnelle américaine (Sacks, Schegloff, Jefferson 1974).

Après avoir exposé notre cadre d'analyse de discours (en particulier les notions de mouvements et de places) (1), nous développons certains aspects théoriques permettant le repérage des voix (2). Différents extraits du même entretien sont ensuite commentés (3), en pointant plus particulièrement les aspects formels présentés en 2 d'un point de vue théorique. Les fonctions interactionnelles et sociales que jouent ces voix sur la scène de l'entretien sont finalement pointées. 


\section{Dialogisme, mouvement (discursif et interprétatif) et place (discursive et sociale)}

Notre cadre d'analyse adopte en arrière-fond certains fondements de la sociologie cognitive (Cicourel, 2002) qui propose de lier l'analyse du contexte au sens large (organisationnel, institutionnel, culturel) à celle du contexte au sens étroit (analysé à partir des interactions locales par une approche d'inspiration conversationnelle et ethnométhodologique) pour insister sur le façonnage réciproque des contextes au sens large et étroit. Notre approche rejoint également certains fondements de la psychologie socioculturelle (Rogoff, 1990 ; Cole, 1994, 1995 ; Wertsch 1991), issus des prolongements de la pensée de Vygotski (1985 [1934]), et dont les liens avec la pensée de Bakhtine ont été soulignés ${ }^{5}$. Ce courant conçoit les processus cognitifs (et notamment les récits de soi) comme (i) distribués à travers les membres d'une communauté et (ii) circulant à travers la culture sous l'influence simultanée des interactions localement accomplies et des cadres socio-culturels d'une époque. Markovà a développé plus globalement en psychologie sociale le concept de "dialogicité » comme "la capacité de l'Ego à concevoir et comprendre le monde dans la perspective de l'Alter et à créer des réalités sociales dans la perspective de l'Alter» (2007 [2003]) : 288).

Notre mode d'analyse mobilise en avant plan l'idée qu'il y a des significations liées aux modes d'enchaînements des énoncés, aux «mouvements » du discours (François, 1984, 1989, 2009 ; Salazar, 1999, 2003, 2008 ; Carcassonne, 2006, 2008).

\subsection{Dialogisme et mouvements (discursifs et interprétatifs)}

Ces mouvements discursifs sont nécessairement liés aux mouvements interprétatifs de celui qui les repère. Le sens «bouge» ou plutôt se «dessine» (François, 1993: 34) entre des énoncés ou des séquences bien délimités parce que le récepteur les met en rapport (de façon plus ou moins obligatoire ou facultative en fonction d'un contexte donné). Les mouvements repérés peuvent correspondre à des mouvements de thèmes, de catégories (en tant qu'aspect plus local d'un même thème), de modes énonciatifs (passer par exemple d'un discours doxique à un discours particulier), de types de discours (passer par exemple d'une argumentation à une narration), etc. Le sens se dessine aussi dans la façon dont ce récepteur peut mettre en rapport ces énoncés ou séquences avec le contexte (large comme étroit) comme avec ses attentes liées au genre ${ }^{6}$ de discours en question.

Tout mouvement de discours repéré par un récepteur au niveau de la production est donc nécessairement un mouvement interprétatif proposé par le récepteur-analyste. Ces deux versants (production/réception) recoupent, sans totalement s'y superposer, une analyse en termes «dialogal/dialogique » (Bres, 2005 : 49) : c'est-à-dire qu'ils permettent d'établir d'une part des liens entre les énoncés co-produits par les interlocuteurs en situation (processus local de co-construction du sens ou « dialogisme interlocutif»), d'autre part des liens avec des discours relevant de sphères dialogiques antérieures ou à venir (« dialogisme interdiscursif $»)$.

Le processus du dialogisme interdiscursif a lieu également avant même la « mise en mots » (François, $1994)^{7}$, le souvenir des événements vécus étant non seulement «transformé » par des lectures ou des récits entendus (interdiscursivité) mais aussi par le processus même de la mémoire qui n'enregistre pas les événements vécus de façon purement objective : "Le souvenir est dans une très large mesure une reconstruction du passé à l'aide de données empruntées au présent, et préparée d'ailleurs par d'autres reconstructions faites à des époques antérieures et d'où l'image d'autrefois est sortie bien altérée.» (Halbwachs, 1950).

La dimension du dialogisme interlocutif est elle aussi plus complexe qu'il n'y paraît, comme l'ont noté Bres \& Nowakowska (2006). Elle implique une modulation des propos de l'interviewé en fonction de l'image qu'il se fait de son interlocuteur ou de ses interlocuteurs (de ses connaissances, de son rôle, etc.). Et cette anticipation incessante de la réponse que le locuteur imagine de la part de son interlocuteur façonne en retour celle de son interlocuteur. De façon plus ou moins consciente, il existe ainsi toujours 
dans tout dialogue une forme de "pré-occupation » (Clot, 1999) des places discursives, en fonction des représentations du rôle de chacun.

\subsection{Mouvements et places (discursives vs sociales)}

Les mouvements discursifs peuvent en effet être vus comme autant de façons d'occuper des places discursives, en tant qu'actualisation concrète des rôles à tenir dans cette situation. Ces places renvoient à la mise en mots des représentations qu'ont les interlocuteurs de la situation, d'eux-mêmes, de l'interlocuteur, de leurs relations réciproques, des thèmes à aborder ou non dans cette situation, d'attendus plus ou moins partagés liés aux statuts des uns et des autres dans un cadre interactif et social donné. Flahaut (1978) et Vion (1999), à la suite de Goffman (1973) ont insisté chacun à leur manière sur le fait que les places se situaient toujours par rapport à un sens pré-attribué mais qu'elles ne se jouaient que dans l'interaction hic et nunc. En d'autres termes, une grande part du sens pré-attribué que les places peuvent approuver ou contrer réside dans les attributs liés aux statuts. Ces attributs (biologiques, sociaux, expérienciaux, de connaissance) impliquent des différences ou des ressemblances entre les interlocuteurs, et par là même un certain nombre d'asymétries ou d'inégalités (Markovà \& Foppa, 1991). Pour Flahaut, toute parole convoque l'interlocuteur à une place corrélative : la distribution des places est tributaire du cadre dans laquelle elle se déploie, et plus largement du contexte social et culturel. La notion de place permet d'articuler ${ }^{8}$ la place de l'interlocuteur dans l'interaction hic et nunc et la place de ce même interlocuteur « dans la formation sociale à laquelle il appartient » (1978 : 138).

Une production discursive s'analyse donc bien au-delà d'un seul acte discursif isolé : elle est vue comme une rencontre entre des énonciateurs ayant des histoires conversationnelles et socio-discursives différentes et dont les traces sont en partie repérables dans la matérialité langagière. Par rapport à un même discours, chacun ne perçoit pas nécessairement le même dialogisme (pris ici comme un ensemble des voix qui le traversent). Une analyse en termes de voix ne saurait être selon nous une analyse en termes de « composantes réelles » mais est toujours une analyse en termes de modes d'interprétation.

\section{Relations entre types de connaissance sur soi, plans énonciatifs et voix}

Nous nous intéresserons ici d'abord aux relations entre voix et types de savoirs sur soi (savoirs transmis vs perçus) (2.1), pour présenter ensuite le jeu discursif entre plans énonciatifs comme un des moyens privilégiés de «marquer » (Bres, 1998) une voix dans un discours (2.2). Les modes de marquage des voix ont été analysés dans des genres de discours très divers et peuvent en effet s'étaler sur un continuum (2.3) allant d'un pôle où la voix est « détachée» du discours de l'énonciateur ((marquage « fort » d'une voix autre) à un pôle où elle y est totalement " intégrée » (marquage faible d'une voix autre)). Les aspects retenus pour l'analyse des extraits présentés en 3 sont ensuite synthétisés dans une grille (2.4).

\subsection{Quelles sont les sources des « connaissances » sur soi ?}

Si un inventaire de ces sources est impossible à constituer de manière exhaustive, nous allons proposer toutefois des axes différenciant ces sources. Le discours que l'on tient sur soi est souvent intermédiaire entre le discours objectif de l'autre et le discours subjectif de soi : tenir un discours sur soi met en effet le locuteur dans la situation d'un quasi-autre qui n'est pas un vrai autre. Toute personne peut parler d'elle en reprenant à son propre compte ce qu'autrui a dit d'elle (par exemple ce qu'on lui a raconté d'elle étant enfant, ce que les médecins disent d'elle) tout en indiquant simultanément ou alternativement ce qu'elle connaît d'elle par familiarité (par exemple la perception qu'elle a de son propre caractère, de son rapport à autrui, mais aussi ses craintes, ses espoirs, etc.). La connaissance de soi par familiarité relève d'un savoir perçu et non transmis par l'autre (et n'est pas toujours explicitée ou mises en mots); elle est cependant souvent étroitement intriquée dans le discours sur soi à une connaissance relevant d'un savoir transmis par l'autre. 
Peut-on qualifier les énoncés relevant du savoir perçu comme un type de voix ? Nous le ferons ici, même si cela peut paraître abusif, en observant qu'il peut y avoir en outre différentes formes d'imbrications dans le discours sur soi entre les savoirs perçus et savoirs transmis :

Parmi les savoirs transmis par l'autre sur soi, nous proposons de distinguer :

- le savoir relevant de la doxa, comme correspondant au discours du « on », au «prêt-à-penser » d'une collectivité. Il s'agit d'un discours «qui circule», comme les proverbes, les formules toute faites, les slogans publicitaires, etc. Husserl a défini ce terme comme un ensemble des croyances et d'idées non objectives. Selon Anscombre (1995: 39), une doxa est déterminée par des topoï, lesquels ont deux caractéristiques centrales. D'une part ce sont des "principes généraux », jamais assertés mais utilisés pour servir «d'appui au raisonnement». D'autre part, «ils peuvent être créés de toute pièce » et sont souvent « présentés comme allant de soi ». Leur existence est un fait de sociologie, variable d'une culture à l'autre ; en outre, comme le résume Sarfati (1997: 33), « deux topoï contraires peuvent coexister dans une même société, au même moment historique (Qui se ressemblent s'assemblent/Les extrêmes s'attirent)».

- le savoir relevant du discours spécialiste, correspondant à la connaissance scientifique. Bakhtine $(1984)^{9}$ a décrit cette connaissance comme abstraite, impersonnelle, entraînant une position " objective » chez celui qui la détient.

- le savoir relevant de l'autre comme correspondant, notamment, au discours rapporté ou au discours de l'autre intériorisé. Bakhtine a souligné à quel point le savoir sur soi-même est « déterminé par le mot réfléchi d'un "étranger"» (Bakhtine, 1970: 268). L'analyse qu'il propose de l'œuvre de Dostoïevski caractérise le discours sur soi comme traversé par le discours moral des autres : «Le héros se juge en fonction de l'idée qu'il se fait d'autrui et de l'opinion sur soi qu'il lui suppose. La conscience de soi est sans cesse doublée par la conscience qu'en a autrui ; le "moi pour moi-même" se réfère constamment au "moi pour les autres. C'est pourquoi le mot du héros sur lui-même se construit sous l'influence incessante du mot d'autrui à son sujet ». (Ibid. : 269). Une conscience de l'autre sur soi, ni totalement intérieure ou extérieure à soi, serait ainsi une autre source majeure du savoir sur soi.

Cette grille de différents types de savoirs sur soi, non exhaustive, peut en outre se décliner plus spécifiquement vis-à-vis du discours médical en distinguant plusieurs types de rapports entre savoirs par familiarité (ou perçus) et savoirs transmis (François, 1995 : 112) :

- celle où les rapports entre les savoirs sont stabilisés. Par exemple, nous savons tous que «le soleil est plus grand que la terre », même si nous ne pouvons pas le percevoir : savoirs perçus et transmis ne se recouvrent pas ici et donc ne s'opposent pas ;

- celle où il y a dominance (du point de vue de l'analyste) de l'un ces savoirs sur l'autre (comme dans "j'ai peur d'avoir un ulcère à l'estomac et c'est le médecin qui me dira si j'en ai vraiment un »). Mais cette opposition entre savoir transmis et savoir perçu (ressenti) ne se résout pas toujours en ayant simplement recours à la parole de « celui qui sait », ce qui amène au troisième cas ;

- celle où les rapports entre ces savoirs ne peuvent pas être stabilisés : c'est ce qui se passe dans l'entretien ici présenté, où la propre voix de la connaissance de soi-même par familiarité (savoir perçu) est constamment mise en regard avec celle du savoir du spécialiste. Comme l'a développé Clavreul: " chacun sait qu'il existe un savoir médical, que personne ne peut prétendre le posséder entièrement, mais qu'il est toujours possible d'y avoir recours. Bien plus, le malade lui-même n'est jamais un autre par rapport à ce discours. Il y participe, il est convié à s'y rallier, à s'y soumettre, et, en fait, il le devance et tente de raisonner sur sa maladie en termes médicaux » (1978: 31).

Cette doxa médicale selon laquelle tout mal aurait une cause est d'ailleurs admise chez tout un chacun, du moins dans la culture occidentale, rejoignant finalement la doxa tout court. Mais, comme le souligne Cicourel dans le cadre de l'analyse du raisonnement diagnostique, «les patients recourent à un champ sémantique particulier ou restreint pour traduire les croyances dont ils font usage à propos de leur maladie - lesquelles croyances, il convient de le souligner, vont souvent à l'encontre du point de vue du 
médecin » (2002: 66). Cicourel analyse le fait que le savoir médical s'insère dans un système symbolique différent de celui des patients, privilégiant un langage abstrait fondé sur un système mnésique externe de savoirs objectivés utilisant des "mémoires externes » (dictionnaires de termes médicaux, manuels de médecine, examens de laboratoires, etc.). Cela nécessite un véritable "recodage » pour le médecin qui « traduit» le discours du patient pour établir son diagnostic. Pour Cicourel, les médecins devraient davantage expliciter ce recodage dans leur communication avec les patients : cela permettrait d'éviter certains malentendus car les patients ne raisonnent pas sur leur maladie uniquement à partir de savoirs objectivés, mais aussi et surtout à partir de savoirs relevant de croyances, lesquelles peuvent être imbriquées à des savoirs plus spécialisés qui circulent dans la vie sociale.

A partir d'une grille synthétisant les propos ci-dessus, nous relèverons dans les extraits de corpus présentés en dernière partie les différentes sources de connaissances sur soi évoquées par l'interviewée, en distinguant (même si elles peuvent parfois se superposer) celles qui relèvent du savoir sur soi

- par familiarité,

- par la doxa,

- par un discours de spécialiste,

- par un discours de l'entourage,

- et les autres (ce dernier tiret nous permet d'insister sur le fait que cette liste ne prétend pas être exhaustive).

Nous croiserons cette première entrée d'analyse avec deux autres aspects formels du repérage des voix : les plans énonciatifs (dont nous présentons les fondements théoriques ci-dessous) et le degré de marquage de la voix comme autre (de fort à faible, cf. 2.3).

\subsection{Voix et plans énonciatifs}

S'il est vrai que l'hétérogénéité (des sources, des sphères de discours, des voix) est toujours constitutive du discours, elle n'est pas toujours montrée : elle n'est dans ce cas pas repérable et aboutit même à une dilution de la notion de dialogisme. Un récepteur ne peut en effet repérer que des voix suffisamment « actualisées » (Bally, 1965 [1934]).

La question des critères permettant de distinguer les plans énonciatifs est importante pour observer les voix dans un discours car ces plans permettent de remonter (en partie) à la source des voix en question. Les marques renvoyant à un plan énonciatif ou à un autre correspondent à une des façons privilégiées de positionner une voix sur le continuum allant de la voix marquée à non marquée (cf. tableau en 2.3).

Benveniste, puis Weinrich ont initialement distingué deux plans énonciatifs, à partir de marqueurs morpho-syntaxiques (tels que les tiroirs verbaux, personnes du verbe, etc.) et lexicaux (tels que les adverbes et locutions temporelles ou spatiales pour l'essentiel) : l'un ancré sur la situation d'énonciation, l'autre renvoyant à une sphère spatio-temporelle déconnectée de la situation d'énonciation.

Weinrich (1989) a repris de manière critique la typologie énonciative «histoire »/« discours» de Benveniste (1966) en introduisant la notion d'affinité entre les termes grammaticaux d'un discours pour distinguer « l'histoire » du « commentaire».

Dans la lignée de ces travaux, et avec une inscription dans le cadre de la théorie culiolienne, DanonBoileau $(1982,1987)$ a décrit comment la prise en compte de marques non seulement grammaticales, mais aussi lexico-aspecto-grammaticales peut contribuer à un "repérage » énonciatif. A partir de là, il a distingué non plus deux « postures énonciatives » comme l'avaient fait Weinrich et Benveniste mais deux « types de repérage » (par deixis, par anaphore) et un « non repérage » par indétermination.

Cette distinction permet de désigner un support pour les modalités appréciatives, évaluatives et autres, tant dans le domaine du « discours » que dans celui de «l'histoire » (ou « récit») ${ }^{10}$. Kuyumkuyam (2006) a proposé plus récemment un aménagement de la dichotomie de Benveniste, en proposant pour sa part de 
considérer le continuum allant de la «mention » au « discours »; la mention correspondant pour elle au domaine élargi du récit et étant vue comme le contraire du « discours»; ce dernier plan restant réservé aux énoncés dont le locuteur est explicité et réfère au sujet parlant.

En s'inspirant de ces différentes analyses, nous examinerons comment les «affinités» (au sens de Weinrich, 1989, et François, $1994^{11}$ ) entre les différents types d'unités lexico-grammaticales permettent de cerner les voix dans un dialogue, en les attribuant à différents plans énonciatifs, définis de la manière suivante :

- le plan du «discours" où les aspects auxquels le discours fait référence sont présentés comme « ancrés » dans la situation d'énonciation ;

- le plan du «récit» où les aspects auxquels le discours fait référence sont présentés comme «désancrés » de la situation d'énonciation (référence future ou passée par rapport au moment de l'énonciation);

- le plan du « commentaire» où les aspects auxquels le discours fait référence sont présentés comme ne renvoyant pas à un moment énonciatif précisément repérable : ce plan peut inclure indifféremment, en la dominant, une énonciation de type discours ou de type récit. Par exemple dans l'énoncé extrait de l'entretien ici présenté, J'me voyais pas, j'voyais pas mon corps euh...maigre, la qualification «maigre » semble avoir été «intégrée » (Grunig \& Grunig, $1985: 25)$ petit à petit. Cette qualification entre en affinité avec d'autres procédés d'objectivation ou de modalités appréciatives qui traversent tout l'entretien (comme le montrent les extraits en III) et confirment cette forme « d'intégration » du discours médical par l'informatrice. Cette intégration est certes peu marquée mais cependant suffisamment actualisée par ces modalités pour être repérée.

De façon plus générale, dans l'ensemble des discours co-construits en entretien, le soi évoqué est un soi qui correspond seulement en partie à l'interviewé, lequel peut être présenté comme étant plus ou moins proche du soi hic et nunc, grâce au jeu sur les frontières entre les différents plans énonciatifs. Ce jeu sur les plans énonciatifs permet de se présenter « soi-même comme un autre » (Ricœur 1990), et permet aussi à un autre pôle, sur une forme de continuum, de présenter l'autre que l'on a été comme étant encore soimême ou en partie soi-même (cf. ex. 1 donné au point 3.2.1). Il montre aussi comment la compréhension de soi-même n'est pas pré-établie mais se « travaille » langagièrement, dans et par l'espace de suspension que constitue un entretien de recherche.

\subsection{Continuum allant d'un marquage "fort » d'une voix autre à un marquage faible}

Quel type de marque signale une voix comme autre? Quelle est place pour cette voix sur le continuum allant du plus marqué au moins marqué ? Bakhtine (1977) a appréhendé le discours d'autrui dans le roman d'une façon originale car il n'a pas répertorié en soi et pour soi les formes du discours rapporté mais les a mises en relation avec le contexte narratif historique. Cela lui a permis de proposer ensuite un classement par périodes du mode d'appréhension du discours d'autrui dans le roman, du plus marqué (« style linéaire ») au moins marqué (« style pittoresque »). Cette terminologie peut-être mise en parallèle avec celle des auteurs dont les noms figurent en gras dans le tableau ci-dessous. Ce tableau ne vise pas l'exhaustivité mais cherche à mettre en évidence (i) la diversité des terminologies et des dialogues étudiés depuis Bakhtine, en particulier autour de cette problématique du marquage des voix ; (ii) le continuum allant du marquage « fort » d'une voix autre (haut du tableau) au marquage le plus faible (bas du tableau). 
Tableau 1 (le continuum est à lire ici de haut en bas)

\begin{tabular}{|c|c|}
\hline $\begin{array}{l}\text { Terminologie renvoyant aux types de } \\
\text { marquage du discours d'autrui, du plus } \\
\text { «fort » au plus discret }\end{array}$ & Genre de discours analysé \\
\hline $\begin{array}{l}\text { Bakhtine }(1977: 161) \\
\text { «style linéaire »: contours extérieurs nets } \\
\text { autour du discours rapporté }\end{array}$ & $\begin{array}{l}\text { romans } \mathrm{du} \text { moyen âge, contexte narratif } \\
\text { relevant } \mathrm{du} \text { « dogmatisme autoritaire » }\end{array}$ \\
\hline $\begin{array}{l}\text { Coste, Mochet, Papo (1986) } \\
\text { discours « attribué » à l'autre par un interviewé }\end{array}$ & entretiens sociologiques \\
\hline $\begin{array}{l}\text { Drew }(1991: 38) \\
\text { énoncés «marqués » par le patient comme } \\
\text { relevant du discours médical }\end{array}$ & $\begin{array}{l}\text { consultations médicales } \\
\text { Patient : I seem to have, what is it - } \\
\text { contracted tendon. } \\
\text { Patient : (...) I was having a problem in the } \\
\text { uh..., I guess } w \text {-what you call the bulk of the } \\
\text { outer uh part of the organ }\end{array}$ \\
\hline $\begin{array}{l}\text { Bakhtine (1977: 162) } \\
\text { "style pittoresque de premier type »: } \\
\text { "gommage délibéré des frontières du discours } \\
\text { rapporté afin de le colorer de son humour, de } \\
\text { son ironie, de sa haine, de son ravissement ou } \\
\text { de son mépris » }\end{array}$ & $\begin{array}{l}\text { romans du } 17^{\mathrm{e}} \text { et } 18^{\mathrm{e}} \text { s., contexte narratif } \\
\text { relevant du «dogmatisme rationaliste » }\end{array}$ \\
\hline $\begin{array}{l}\text { Coste, Mochet, Papo (1986: 72) } \\
\text { " citation emblématique d'attitude »: } \\
\text { "discours hypothétique imaginé, l'énoncé cité } \\
\text { étant choisi pour sa valeur évocatrice d'une } \\
\text { attitude, pour ce qui est dit, mais plus encore } \\
\text { pour ce qui est manifesté » }\end{array}$ & entretiens sociologiques \\
\hline $\begin{array}{l}\text { Mochet (1989: 185) : citations emblématique } \\
\text {-citation d'un discours tenu (présenté comme un } \\
\text { discours rapporté) } \\
\text {-citation d'un discours de la pensée } \\
\text { - citation d'un discours potentiel, représenté, } \\
\text { imaginé }\end{array}$ & $\begin{array}{l}\text { entretiens de recherche (extrait du corpus ici } \\
\text { présenté) } \\
\text { - euh, j'ai dit à maman écoute hein je } \\
\text { préfère mourir que d'revivre ça } \\
\text { - je me suis dit maintenant, si jamais je } \\
\text { retombe malade, je préfère mourir que de } \\
\text { revivre ça } \\
\text { - ma copine va encore me dire que c'est une } \\
\text { mauvaise idée }\end{array}$ \\
\hline $\begin{array}{l}\text { Bakhtine }(1977: 162) \\
\text { «style pittoresque de second type »: «quasi- } \\
\text { absorption du contexte narratif par le discours } \\
\text { rapporté » et une "infiltration du discours } \\
\text { rapporté par les répliques et commentaires de } \\
\text { l'auteur» }\end{array}$ & $\begin{array}{l}\text { romans de la fin du } 18^{\mathrm{e}} \text { et } \mathrm{du}_{19^{\mathrm{e}} \mathrm{s} ., \text { contexte }} \\
\text { narratif relevant du «l'individualisme } \\
\text { réaliste et critique » }\end{array}$ \\
\hline $\begin{array}{l}\text { Bakhtine }(1977: 162) \\
\text { « dilution du contexte narratif » }\end{array}$ & $\begin{array}{l}\text { romans de l'époque } \\
\text { caractérisés par un } \begin{array}{l}\text { contemporaine, } \\
\text { "individualisme }\end{array} \\
\text { relativisé » }\end{array}$ \\
\hline $\begin{array}{l}\text { Grunig et Grunig }(1985: 25) \\
\text { «énoncés intégrés »: «tout ce qui est venu } \\
\text { s'inscrire dans un individu, en ayant sa source } \\
\text { dans les paroles, les énoncés des autres » }\end{array}$ & tout type de discours \\
\hline $\begin{array}{l}\text { Authier-Revuz }(1982: 146 ; 1894,1995) \text { : } \\
\text { hétérogénéité constitutive }\end{array}$ & tout type de discours \\
\hline
\end{tabular}




\section{Commentaires du tableau 1 :}

- Pour renvoyer aux contours extérieurs nets autour du discours rapporté dans les romans du Moyen Age, Bakhtine introduit l'expression «style linéaire ».

- On retrouve dans les exemples de Coste et Drew un «style linéaire » selon la terminologie de Bakhtine : Coste et al. remarquent de façon classique dans des entretiens sociologiques que l'interviewé peut «attribuer » un discours à quelqu'un d'autre ; de façon proche, Drew note dans certains entretiens médicaux l'utilisation d'une terminologie médicale par les patients, avec simultanément le fait qu'ils ne s'attribuent pas la source de ce savoir (les procédés utilisés par les patients cités par l'auteur pour dissocier les sources énonciatives sont soulignés dans les extraits joints dans le tableau 1).

- Pour renvoyer au «gommage délibéré des frontières du discours rapporté » dans les romans du $17^{\mathrm{e}}$ et $18^{\mathrm{e}} \mathrm{s}$., Bakhtine utilise l'expression « style pittoresque de premier type ».

- De façon un peu plus avancée vers le pôle du non marquage sur notre continuum, Coste et al. introduisent les «citations emblématiques d'attitude», qui confèrent selon nous un caractère argumentatif au discours dans lequel elles s'insèrent. Mochet propose ensuite de distinguer plusieurs types de citations «emblématiques d'attitude» en les caractérisant par le fait qu'elle «traduisent l'esprit » de ce qui a été dit et par là même l'interprètent.

- Sur ce continuum, on trouverait ensuite ce que Bakhtine appelle «style pittoresque de second type », typique les romans de la fin du $18^{\mathrm{e}}$ et $\mathrm{du} 19^{\mathrm{e}} \mathrm{s}$., avec une «infiltration du discours rapporté par les répliques et commentaires de l'auteur »; puis les romans de l'époque contemporaine caractérisés par une « dilution du contexte narratif ».

- Se trouvent ensuite sur ce continuum ce que Grunig et Grunig appellent « énoncés intégrés », lesquels sont parfois (mais pas toujours) suffisamment actualisés pour être repérables (cf. exemple commenté cidessous au point 2.3).

- Enfin, à l'extrémité du continuum, se trouveraient les voix non marquées, donc non actualisées et non repérables (« hétérogénéité constitutive » de tout discours, Authier-Revuz, 1982, cf. note 2).

\subsection{Grille synthétique de l'analyse des voix dans un discours}

L'ensemble des fondements théoriques exposés dans cette partie peuvent être regroupés dans une grille permettant de synthétiser les différentes entrées que nous allons adopter pour analyser des extraits de corpus dans la partie 3 .

Cette grille rappelle les aspects développés ci-dessus en 2 (aspects formels du repérage des voix) et ajoute une interrogation quant aux fonctions jouées par ces voix sur la scène de l'entretien :

Tableau 2

\begin{tabular}{|c|c|c|c|}
\hline \multicolumn{3}{|c|}{ Aspects formels du repérage des voix } & Fonctions des voix \\
\hline $\begin{array}{l}\text { Type de } \\
\text { connaissance } \\
\quad \text { sur soi } \\
\text { - par familiarité } \\
\text { - doxa } \\
\text { - spécialiste } \\
\text { - entourage } \\
\text { - autres types de } \\
\text { connaissances } \\
\text { (cf. point 2.1) }\end{array}$ & $\begin{array}{l}\text { Plan énonciatif } \\
\text { - récit } \\
\text { - discours } \\
\text { - commentaire } \\
\text { (cf. point 2.2) }\end{array}$ & $\begin{array}{l}\text { Degré de marquage } \\
\text { de la voix comme } \\
\text { autre (de fort à faible) } \\
\text { Quel type de marque } \\
\text { signalant la voix } \\
\text { comme autre? } \\
\text { Quelle place pour cette } \\
\text { voix sur le continuum } \\
\text { allant de marqué à non } \\
\text { marqué? } \\
\text { (cf. point } 2.3 \text { ) }\end{array}$ & $\begin{array}{l}\text { - Argumentative? } \\
\text { - Construction } \\
\text { d'une image } \\
\text { positive de soi? } \\
\text { - Renversement } \\
\text { des places } \\
\text { sociales? } \\
\text { - Autres? }\end{array}$ \\
\hline
\end{tabular}




\section{Commentaires d'extraits véhiculant plusieurs voix explicatives de la maladie dans l'entretien de F.}

Nous allons donc commenter certains extraits de corpus (3.3) à partir de la grille présentée en 2.4, après avoir présenté les conditions de recueil de ce corpus et ses implications dialogiques (3.1) et insisté sur la multiplicité d'explications causales proposées par l'interviewée pour expliquer l'origine de sa maladie (3.2).

\subsection{Présentation du corpus: conditions de recueil, implications dialogiques, choix de l'entretien de $F$.}

L'entretien choisi fait partie d'un corpus plus important (une quinzaine d'entretiens) mené auprès de personnes volontaires présentant des addictions variées, mettant en jeu leur vie. Les interviewers de cet ensemble d'entretiens, tous psychiatres ou psychanalystes, cherchaient à comprendre la «conduite ordalique », définie ainsi : "répétition d'une épreuve comportant un risque mortel, dans laquelle le sujet s'engage afin, par sa survie, de prouver sa valeur intrinsèque, ainsi reconnu par les puissances transcendantes du Destin » (Charles Nicolas \& Valleur, 1982 : 90).

Comme l'expose Valleur, l'addiction comporterait à la fois un versant de désubjectivation et de passivité, à travers la dépendance, et une quête de sens par son versant ordalique, lequel constituerait la face activement transgressive de la conduite. Les conduites ordaliques seraient ainsi «des tentatives, pour le sujet dépendant, ayant perdu le contrôle de sa vie (...), de reprendre en main son destin. Ces conduites constitueraient alors l'envers de la dépendance » (Carcassonne \& Valleur, 1997 : 91-92).

Partant de cette hypothèse, les chercheurs avaient prévu d'aborder, au cours d'entretiens enregistrés, menés de façon semi-directive, les thèmes suivants : risque activement recherché et vécu positivement, conduites associées participant au versant ordalique, psychotraumatismes et ruptures précoces, vécu de ces événements. Les interviewés, tous volontaires, avaient été informés avant l'entretien par un préentretien oral et par une lettre écrite décrivant les objectifs de la recherche, résumée en ces termes : «mener un travail de recherche autour d'événements de vie (deuil, perte, mort) et du rapport au risque (toxicomanie, consommation abusive de médicaments psychotropes, troubles des conduites alimentaires, tentative de suicide, etc. " (Valleur, 1994 : 182).

Parmi les multiples implications dialogiques liées au contexte large et étroit (Cicourel, 2002, cf. point 1) de ces corpus, nous pouvons citer les suivantes : les discours des interviewés ont nécessairement été «pré-orientés » par cette lettre comme par le pré-entretien oral de présentation de la recherche; simultanément les hypothèses de recherche ont nécessairement influencé les interviewers dans leurs façons de réagir aux propos des interviewés et plus globalement de jouer leurs rôles d'interviewers. Ce dialogisme interdiscursif se double au niveau du dialogisme interlocutif par le fait que les interviewés savaient que leurs propos allaient être objet de réflexion au sein d'une équipe de recherche constituée de destinataires « à l'horizon » (Gadamer,1960; Jauss, 1978) ${ }^{12}$ du dialogue co-construit hic et nunc.

L'analyse psychopathologique de ces entretiens, subventionnée par le ministère de la recherche, a donné lieu à un rapport de recherche (Valleur, 1994) ${ }^{13}$. Nous avons participé à ce rapport en analysant certains des entretiens d'un point de vue linguistique ${ }^{14}$ et poursuivi l'analyse de ces entretiens ${ }^{15}$ en mettant notamment en évidence des différences de «micro-genre» (Carcassonne, 2008) dans le discours des informateurs : très interprétatif chez F., plus factuel chez les autres. Nous avons soulevé l'hypothèse d'un lien entre la spécificité du maniement du micro-genre interprétatif chez F. dans ce type de situation et certains éléments de sa vie :

a- F. a la conduite la moins ordalique : c'est la seule à ne pas avoir d'addiction vis-à-vis d'un produit (même si l'anorexie est considérée comme une addiction sans produit, cf. Goodman, 1990, Bailly et al., 1991, Mc Dougall et al., 2001). 
b- Elle est la seule à travailler dans le cadre d'un mi-temps thérapeutique (elle a été anorexique durant une quinzaine d'années avant de devenir boulimique et explique au cours de l'entretien qu'elle a pu s'en sortir grâce à la reprise de son travail).

c- Elle réfléchit depuis de longues années en psychothérapie sur son histoire personnelle et sur les causes éventuelles de sa maladie.

La pratique régulière du micro-genre interprétatif en psychothérapie faciliterait la production (Schneuwly, 1994, cf. note 6; Thomassin, 1999) de ce même micro-genre en entretien de recherche. Ce micro-genre donnerait, grâce au maniement des procédés méta-communicatifs (minimisation, modalisation, polyphonie), la possibilité de prendre du recul face à l'épreuve ou la souffrance. Vion et al. (2001) ont en effet noté que cette possibilité semblait aller de pair avec la capacité de se mettre à la place de l'autre l'interlocuteur - au plan interlocutif comme au plan de l'intersubjectivité.

Dans la mesure où ce résultat peut être intéressant pour la pratique des soignants, nous avons justement choisi d'approfondir ici la façon dont F. raisonne sur sa maladie (au sens de Cicourel, 2002), en particulier dans sa façon de convoquer différents types de voix dans son discours.

\subsection{Une multiplicité d'explications causales proposées par l'interviewée pour expliquer l'origine de sa maladie :}

\subsubsection{Cinq causes possibles : quelques exemples}

Sur l'ensemble de l'entretien, F. propose en fait cinq explications différentes des causes possibles de l'anorexie : avoir voulu maigrir pour être plus féminine, avoir voulu attirer l'œil des autres, avoir un « rapport anal» important, avoir une mauvaise relation avec ses parents, avoir une prédisposition. Ces causes sont présentées comme étant toutes plausibles, mais elles sont toujours finalement mises en regard avec la dernière (une prédisposition), présentée comme étant la plus plausible. Avant de nous focaliser sur ces deux dernières causes (3.3), nous donnons ici quelques exemples de la façon dont certaines autres causes sont présentées.

La première (vouloir être plus féminine) relève d'un savoir perçu plus que transmis. F. la présente comme une explication très incertaine (qu'il s'agisse de son personnage au passé ou au présent), c'est-à-dire de façon tout aussi modalisée dans les deux plans énonciatifs du récit et du discours (« je croyais »/ «je crois »), comme nous le montre le rapprochement à distance entre deux groupes d'énoncés (28-31 et 853$855)^{16}$ :

\section{Exemple 1}

28 donc j'étais très masculine.

29 Je croyais qu'cétait parce que j'aimais

30 et finalement en moi même je me suis révoltée contre ça

31 parce que finalement j'étais très féminine au fond de moi-même.

853 donc j'étais très musclée, très masculine.

854 Et ça je crois qu'ça plaisait pas aux hommes

855 C'est pour ça que j'ai voulu changer

Lorsqu'elle évoque la cause du « rapport anal », F. semble alors faire référence au « stade oral » de Piaget (qu'elle caractérise par un besoin de «mettre des choses dans la bouche»), dans une forme de lapsus. F. reprend ici à son compte un terme spécialisé de l'autre (savoir transmis) sans le maîtriser, en l'imbriquant fortement à du savoir perçu : 


\section{Exemple 2}

1424 Comment vous vous expliquez ce comportement particulier par rapport à la nourriture qui tenait beaucoup de place en fait?

1425 je sais pas pourquoi

1426 et en plus pourquoi j'ai voulu en faire ma profession?

$142 y$ a quand même un lien

1428 je crois que le rapport anal est très important

1429 d'une part j'ai sucé mon pouce jusqu'à 12 ans

1430 j'arrivais pas à me passer de mon pouce

1431 et j'ai et maintenant pourquoi je fume

1432 c'est pas à cause de la nicotine

1433 c'est pour avoir quelque chose à ma bouche

1434 parce que je pouvais très bien fumer des LTB les cigarettes en pharmacie là

1435 j'aime les autres parce que c'est plus fort

1436 mais moi ça me gêne pas

1437 donc c'est le signe que j'ai besoin de quelque chose dans la bouche

1438 quand je fume pas qu'est ce que fais je mange des bonbons

1439 il faut que j'aie toujours quelque chose à la bouche

1440 et voyez quand j'ai maigri je mangeais pas mais je mangeais des bonbons

1441 donc le besoin anal doit être important chez moi quelque chose à manger le contact la bouche

F. avoue ne pas connaître l'explication demandée par son interlocuteur (1424) pour prendre ensuite ellemême la place du questionneur (1426) et pointer un éventuel lien entre sa profession (diététicienne) et son trouble du comportement alimentaire (1427). Le connecteur «quand même» a une valeur de modalisation et actualise implicitement le contre-argument selon lequel il n'y aurait pas de lien. L'explication du « rapport anal» est elle aussi introduite par une modalisation («je crois »), en affinité avec les énoncés 1426 et 1426 qui indiquent par d'autres moyens (négation, question) que F. n'est pas certaine des explications qu'elle avance. Cette explication est ensuite illustrée par des exemples expérientiels concrets passés ou actuels (avoir sucé son pouce, fumer, manger des bonbons), ce que F. généralise en 1439 dans un temps de l'habituel en rappelant son hypothèse d'un « besoin anal » en 1441.

\subsubsection{La cause « les parents » est présentée comme partagée par l'interlocuteur}

F. sait que son psychothérapeute et son interviewer se connaissent et sont tous deux impliqués dans la recherche en question : elle mentionne deux fois son nom au cours de l'entretien («Docteur S.»), en indiquant au passage qu'elle recherche les causes de sa maladie dans le cadre de cette psychothérapie (584-585), activité qu'elle qualifie de « difficile» (ce que nous pouvons mettre ici en lien avec la notion de « difficile à dire », Gardin, 2006) :

\section{Exemple 3}

584 d'ailleurs avec le Docteur S. on parle souvent de, parce que j'ai toujours pas trouvé l'origine vraiment de l'anorexie

585 et on parle souvent de mes parents, de mes rêves

Elle indique lors de la deuxième mention du Docteur S. que cette recherche de causalités se fait en particulier en creusant le « rapport vis-à-vis de ses parents » (2170-2175) :

2170 je comprends que pour vous l'essentiel c'est le rapport vis-à-vis de mes parents

2171 mais je peux pas en parler beaucoup parce que c'est un peu le travail que je fais avec le docteur $S$.

2172 et j'arrive pas à à cerner la relation c'est très difficile

2173 ça reste un, ça reste vraiment c'est trop loin et c'est trop compliqué

2174 et les parents étaient avec moi pendant vingt ans ils ont été avec moi 


\section{5 je peux pas savoir à quel moment il y a eu des problèmes à quel moment c'est très difficile}

F. semble considérer durant tout l'entretien que son interviewer M. et le Docteur S. partagent le même point de vue selon lequel « les parents » peuvent être une piste de recherche pour comprendre l'origine de l'anorexie. Or M. n'explicite à aucun moment son propre point de vue sur cette question. Il demande même à $F$. (deux fois au cours de l'entretien) de justifier cette position :

\section{Exemple 4}

1693 ce qui est difficile c'est qu'ils [mes parents] sont sans doute la cause de mon anorexie

1694 mais ils sont en même temps la cause de de ma guérison

1695 donc je peux pas leur en vouloir hein

1696 ils ont fait leur rôle

1697 ils ont ils sont au contraire moi je trouve que j'ai eu des parents très disponibles hein

1698 et en quoi vous pensez qu'ils sont la cause?

1699 ben j'en sais rien

1700 tout le monde dit ça

1701 c'est vrai on m'dit toujours que...

1702 non mais vous-même?

1703 non non je leur reproche rien à mes parents (...)

Vers la fin de l'entretien, F. dira encore : Je comprends que pour vous l'essentiel c'est le rapport vis-à-vis de mes parents (énoncés 2170-5).

La diversité des explications proposées par F. au cours de l'entretien montre plus globalement qu'elle vit cet entretien comme une recherche de causalité, introduisant d'emblée la doxa médicale (occidentale) selon laquelle un phénomène pathologique doit avoir une cause. En utilisant des termes spécialisés («besoin anal», ici de façon inadéquate) et en présentant certaines explications relevant de la doxa médicale (qu'elle présenter comme nécessairement partagée), elle semble chercher à établir une forme de connivence avec son interlocuteur. Nous allons voir dans les extraits présentés en 3 qu'elle cherche surtout à lui proposer une autre explication, ne relevant pas de la doxa mais de son savoir « perçu » et qu'elle va finalement renverser en partie l'asymétrie attendue dans ce type d'entretien pour raisonner «à égalité » avec le médecin sur sa maladie.

\subsection{Analyse d'extraits}

La comparaison des extraits choisis, produits à différents moments dans l'entretien de F., met en évidence le mouvement discursif et argumentatif suivant : pour nuancer un discours présenté préalablement comme doxique ( $1^{\mathrm{er}}$ extrait), F. commence par prendre quelques précautions (deuxième extrait) semblant en cela vouloir ménager les convictions de son interlocuteur. Le point de vue est d'abord introduit comme une hypothèse par le biais de marques métadiscursives. Dans un second mouvement discursif (troisième extrait), F. réaffirme son argument mais n'utilise plus les marques de précautions notées dans le deuxième extrait. Ce mouvement d'assurance dessine presque une négation du rôle des parents dans l'anorexie. Tout au plus ce rôle est-il admis comme une concession faite à l'interlocuteur.

Nous allons constater dans ces trois extraits représentatifs de l'ensemble de l'entretien une orchestration de différentes voix, avec une forme de mise en tension de ces voix par le biais du mode énonciatif intriquant la voix de « je » (discours sur soi par «familiarité », « savoir perçu») et la voix de l'autre (savoir transmis), en l'occurrence ici majoritairement celle du discours médical.

\subsubsection{Premier extrait}

L'entretien de F débute ainsi :

1 j'ai commencé par faire de l'anorexie très grave à l'âge de 14 ans juste après ma puberté

2 bon l'origine exacte je la cherche encore actuellement 
3 je pense qu'il y a un conflit comme toujours avec les parents

4 mais surtout je n'aimais pas mon corps

En 1, l'affinité entre l'évaluation « très grave » et la mention temporelle « juste après ma puberté », font résonner le genre discursif « anamnèse médicale », et donc une voix de spécialiste. Cette voix est portée en même temps par une forme de mise en scène de soi dans la mesure où $F$. montre à son interviewer comme à ceux qui liront plus tard ses propos transcrits qu'elle est capable de parler d'elle comme un médecin parlerait d'elle.

En 2, est initiée la voix de la doxa médicale qui va traverser l'ensemble de l'entretien, avec l'expression «l'origine exacte », désancrée au niveau énonciatif.

En 3, avec l'expression « je pense », le plan énonciatif de type discours continue à être dominant tandis que se poursuit le mouvement de désancrage énonciatif amorcé en 2 , véhiculant quant à lui la voix de la doxa médicale. Ce désancrage est repérable à partir d'un certain nombre d'éléments qui introduisent une énonciation de type commentaire dans celle du type discours, à savoir : le présentatif il y a, le terme anorexie, l'absence de possessifs, l'expression comme toujours.

Ces éléments donnent au contenu de cet énoncé une portée de vérité générale, F. se présentant comme adhérant à cette voix générique.

Avec l'utilisation de l'indéfini « un » (« un conflit») et le verbe de discours introducteur « je pense », la double voix de la doxa médicale (intermédiaire entre le discours du « on » et le discours du spécialiste) se teinte également de la voix individuelle de F. (comme l'a noté Martin (1983: 155), «l'énoncé universel construit avec « un » regarde souvent du côté de l'expérience particulière. Si l'on dit qu'un bon vin ne fait pas de mal, on a en vue par exemple, l'inférence que la bouteille de vin que je vous offre ne vous fera pas mal ${ }^{17}$ ).

En 4, F. se présente non seulement comme quelqu'un qui est capable de faire siennes les théories «psy », mais aussi de les moduler, comme nous le montre le marqueur «mais surtout» qui introduit une autre explication de l'origine de l'anorexie, relevant cette fois-ci d'une source appartenant à la voix de son « personnage » au passé, lequel est actualisé dans une énonciation de type récit.

Cette voix est celle de la sphère de la connaissance de soi «par familiarité »; elle est présentée ici comme un argument supérieur au précédent pour expliquer l'origine de l'anorexie.

\subsubsection{Deuxième extrait}

Un peu plus tard dans l'entretien $\mathrm{F}$ est amenée à dire qu'elle est venue à Paris faire ses études de diététicienne :

105 j'suis partie à Paris, donc mes parents me surveillaient plus

106 c'était un moyen de leur échapper aussi à mes parents qui étaient quand même très présents dans ma maladie.

108 ils étaient très présents dans votre maladie?

109 oui c'est sûr que ma mère était très effrayée

110 parce qu'on sait que dans l'anorexie mentale y a toujours un conflit avec les parents

111 ça ils le savaient

112 ils étaient pas

113 on a eu beaucoup d'entretiens avec les psychiatres

114 et ma mère savait qu'il y avait un rapport mère enf-mère enfant qui n'était pas résolu

115 c'est vrai qu'ma mère était très autoritaire

116 moi c'est la question que je me suis posée de des

117 mes parents ont eu quatre enfants j'étais la troisième

118 on a tous eu la même éducation

119 mes parents étaient très rigoureux là-dessus

120 l'un n'avait pas plus que l'autre 
121 donc j'me suis toujours posé la question

122 pourquoi moi j'étais malade alors que ma sœur et mon frère qui ont eu exactement les mêmes conditions d'éducation que moi n'ont pas été malades

123 bon finalement c'est pas rien que la faute de mes parents

124 c'est aussi une prédisposi une prédisposition

125 parce que mes parents ma sœur aurait très bien pu être aussi malade si comme on a eu la même éducation

F. présente dans cet extrait deux causes éventuelles de l'anorexie : ses parents et une prédisposition.

\section{- Première cause : les parents}

Cette cause est présentée d'abord comme relevant d'un discours transmis par un « on » indéterminé en 110 (parce qu'on sait que dans l'anorexie mentale, y a toujours un conflit avec les parents). Cet énoncé reprend très nettement à distance l'énoncé 3 je pense qu'il y a un conflit comme toujours avec les parents.

Cependant, le « conflit des sources » qui existait dans cet énoncé 3 entre je pense (discours du « je ») et comme toujours (discours doxa médicale) n'apparait plus dans cet énoncé 110 : ici, la source est donnée uniquement comme étant le discours du «on», avec l'utilisation de "on sait que ». L'énoncé 111, thématisation du propos précédent par le biais de la reprise anaphorique «ça» (ils le savaient) a pour effet d'insister sur le fait que F. accepte ce discours du « on » même si elle va le nuancer.

La cause « les parents » (comme étant à l'origine de l'anorexie) est ensuite présentée comme relevant d'un discours transmis par les psychiatres : on a eu beaucoup d'entretiens avec les psychiatres et ma mère savait qu'il y avait un rapport mère enf/ mère enfant qui n'était pas résolu.

On note un léger déplacement catégoriel dans le sens où après avoir invoqué ses parents comme se sachant être la cause de l'anorexie en 110, F. n'invoque plus que sa mère en 114. Dans les deux cas, c'est le discours rapporté de l'autre comme sachant qui fait le lien entre le discours expérientiel de soi et le discours générique de "on". A cette cause (qui reprend la voix du discours médical et relève donc du savoir transmis), F. en ajoute ensuite une autre relevant de sa connaissance d'elle-même "par familiarité », celle de la prédisposition.

\section{- Seconde cause : une prédisposition}

F. prend de toute évidence des précautions pour invoquer cette cause plus personnelle. Elle ne donne pas tout de suite son point de vue mais le présente sous forme de question (énoncé 122), laquelle est introduite par deux énoncés métadiscursifs en 116 et 121 :

116 moi c'est la question que je me suis posée de des

121 donc j'me suis toujours posé la question

122 pourquoi moi j'étais malade alors que ma sœur et mon frère qui ont eu exactement les mêmes conditions d'éducation que moi n'ont pas été malades

L'adverbe toujours de l'énoncé 121 présuppose que F. n'a pas de réponse à cette question. Cette question peut-être qualifiée de "citation emblématique d'attitude » (cf. définition au point 2.3). F. présente au préalable l'arrière-fond à connaître pour comprendre la question qu'elle s'est posée à elle-même (énoncés 117 à 120) :
117 mes parents ont eu quatre enfants j 'étais la troisième
118 on a tous eu la même éducation
119 mes parents étaient très rigoureux là-dessus
120 l'un n'avait pas plus que l'autre
123 bon finalement c'est pas rien que la faute de mes parents
124 c'est aussi une prédisposi- une prédisposition

En 123 et 124, F. donne une réponse à la question qui était présentée comme n'ayant pas de réponse en 121 et 122. La juxtaposition de ces deux groupes d'énoncés implicite qu'il s'agit d'une «éventuelle » réponse à cette question. Le discours particulier et expérientiel précédent (117-122) aboutit ici à une 
conclusion de source personnelle qui s'oppose au discours savant des psychiatres (présenté en 110-114). La rapidité de cette alternance entre ces différentes voix dessine une explication caractérisée par son mode de pulsation : c'est ici l'analyse des mouvements énonciatifs, et non des temps grammaticaux, qui permet de caractériser le discours de F.

Les termes introducteurs bon et finalement sont en affinité pour introduire la « reconsidération » de F. par rapport au jugement énoncé précédemment et présenté comme relevant de la doxa («j'ai fait une anorexie à cause de mes parents ») : ils jouent tout autant un rôle de connecteur argumentatif que de modalisation. F. effectue donc ici une remise en cause de la doxa, mais de manière timide. D'une part, cette remise en cause est présentée comme une éventuelle réponse à une question. D'autre part, mis à part les termes introducteurs bon finalement, cette remise en cause ne s'accompagne pas de marques énonciatives explicites (du type " je pense que ») : c'est le mouvement discursif entre les énoncés précédents (116 à 122) et ceux-ci (123 et 124), qui permet de déterminer la source énonciative de cette remise en cause. En 125, F. explicite le mouvement de pensée qui lui permet de modifier le jugement présenté préalablement comme doxique :

\section{5 parce que mes parents ma sœur aurait très bien pu être aussi malade si, comme on a eu la même} éducation.

Cet énoncé 125 reprend à distance les énoncés 118,120 et 122 : un effet d'insistance est obtenu et renforcé (de façon discrète) par les modalisations (conditionnel de aurait, adverbe bien) qui sont renforcées par l'instensificateur très.

L'énoncé 115 C'est vrai qu'ma mère était très autoritaire, dans lequel F. formule également un jugement en biffant la source énonciative de l'énoncé renforce le constat selon lequel F. explicite plus facilement les sources énonciatives relevant de la doxa (énoncé 110) que celles relevant de «je». Salazar \& Grossen (2008) ont fait la même remarque à propos d'autres information en situation d'entretiens cliniques : ce phénomène peut s'expliquer ici par le fait que F. essaye de ménager ce qu'elle croit être les convictions de son interlocuteur, ou par le fait au contraire qu'elle cherche à proposer une forme de mise en scène positive d'elle-même montrant son savoir médical.

\subsubsection{Troisième extrait}

Plus tard dans l'entretien, F. va être plusieurs fois en continuité sur elle-même à distance pour avancer sa « propre version » de l'origine de l'anorexie. Elle va prendre en même temps une certaine assurance pour critiquer la doxa médicale et marquer sa voix de la connaissance d'elle-même par familiarité comme plus adéquate pour expliquer l'origine de son anorexie.

Dans ce deuxième extrait, on constate un mouvement argumentatif similaire au niveau du contenu à celui qui vient d'être commenté.

169 mais souvent j'avais une sorte de nostalgie pendant les vacances

170 je m'ennuyais

171 j'disais à maman " maman j'm'ennuie »

172 elle me dit " pourquoi »

173 "parce que j'suis pas à l'école on fait toujours la même chose »

174 et j'sais pas je sais pas si c'était les parents qui....

175 ou c'était mon état d'âme personnelle

176 je sentais que j'avais un certain blues dans l'esprit qui n'allait pas

177 et après j'suis tombée malade et j'ressens un peu les mêmes impressions

178 donc je pense qu'il y a quand même

179 bon y a l'éducation d'mes parents

180 mais y a quand même une personnalité personnelle en moi quelque chose de profond en moi qui a fait que j'ai fait cette maladie. . 
A nouveau, F. admet la « doxa » médicale selon laquelle ses parents seraient à l'origine de son anorexie (en 174) pour présenter ensuite sa propre explication (178-180), explication relevant d'une connaissance d'elle-même perceptuelle et expérientielle (176-177), d'un savoir perçu et non transmis.

On note cependant des différences dans la présentation des arguments :

a- la doxa médicale est moins vite admise. En effet, le rôle des parents est d'abord présenté de manière très hypothétique en 174 pour être ensuite admis comme une concession en 179, ce qui est marqué par le biais du « connecteur-modalisateur » "bon" (utilisé par contre d'une façon très proche de l'énoncé 123 commenté en 3.3.2);

b- la propre version de F. est présentée de manière logique et « objectivée » grâce à l'effet produit par le connecteur « donc » en 179.

c- le savoir sur soi par familiarité (l'ennui) est véhiculé par des citations emblématiques d'attitudes (171, 173) présentées comme des réponses à une question de la mère («pourquoi »), évoquant au présent un dialogue passé : ce procédé renforce l'effet d'orchestration des voix dans le discours de F.

La présence du connecteur « mais » et de la modalisation « quand même » sont en affinité pour donner à l'énoncé de F. une valeur de contre-argument par rapport à la doxa médicale. Ils marquent la source de cette voix comme relevant d'un savoir perçu et non transmis, cela dans un plan énonciatif intermédiaire entre le discours et le commentaire (du fait d'un relatif désancrage énonciatif avec le présentatif «y a » et le présent générique).

En contre-argumentant par rapport à ce qu'elle croit être une doxa médicale, F. semble vouloir tout à la fois ménager les convictions de son interlocuteur, et montrer une image de quelqu'un qui possède ce savoir.

Sur l'ensemble de ces trois extraits, F. propose donc des arguments et des contre-arguments pour justifier le fait que ses parents ne sont pas les seuls en cause dans l'apparition de son anorexie. Elle invoque ses parents comme une étant une cause éventuelle dans un mouvement discursif assez particulier: elle présente d'abord ce fait comme relevant de la doxa, c'est-à-dire comme un fait que tout le monde connaît, y compris son interlocuteur. Puis tout en reprenant à son compte cet argument, elle y apporte une première nuance qui pourrait se gloser ainsi : «il n'y a pas que mes parents qui sont à l'origine de l'anorexie mais aussi une prédisposition personnelle». Cet argument personnel est justifié au préalable par un discours particulier et expérientiel. Au discours transmis relevant de la doxa médicale (110-114, 174, 179), F. oppose un discours de source personnelle (123-124,180), lequel est justifié par un discours particulier et expérientiel relevant d'un savoir sur soi «par familiarité » (117-122, 169-177), que nous considérons comme une voix parmi d'autres.

\section{Conclusion}

F. a donc une façon particulière de raisonner sur sa maladie en utilisant différentes voix lui permettant tout à la fois de s'expliquer et de se valoriser. En étant étendu à des corpus plus importants, ce type d'analyse peut constituer un éclairage utile non seulement pour les soignants mais pour tout chercheur se questionnant sur la façon dont un interviewé se positionne par rapport à d'autres voix dans un entretien de recherche, d'un point de vue discursif comme social.

Tout au long de l'entretien, F. cherche une cause à sa maladie tout en remettant en question l'idée qu'il puisse y en avoir une, en particulier celle qui impliquerait ses parents. Concernant spécifiquement la cause des parents, nous avons noté une fluctuation très nette dans la façon dont $F$ la ratifie : elle peut être, selon les moments de l'entretien, présentée comme admise et partagée par tous ou être remise en cause. F. avance alors pour cela un «savoir sur soi par familiarité » (ou "perçu»), tout en prenant certaines précautions pour ménager la face de son interlocuteur (alors même que son interviewer n'indique à aucun moment qu'il adhère à cette explication de l'anorexie). F. se présente ainsi comme quelqu'un qui sait adopter une position critique par rapport au savoir transmis (relevant du discours médical et plus généralement des autres). Ses différentes propositions d'interprétation du comportement anorexique 
montrent à l'interlocuteur, lui-même médecin et chercheur, une prise en compte de ses "préoccupations » d'interviewer.

La capacité de F. à convoquer des voix et à savoir se mettre à la place de l'autre peut être liée à sa grande expérience de la psychothérapie. Elle en retirerait une certaine aisance à parler d'elle-même, à évoquer différentes hypothèses sur l'origine de sa maladie: mettre en mots les arguments de l'autre pour les opposer aux siens propres lui permettrait de proposer la voix de la connaissance sur soi par familiarité (savoir perçu) comme une voix tout aussi plausible que la voix des autres, qu'ils soient experts ou non.

Simultanément, la multiplicité des explications causales proposées par F. pour tenter d'expliquer l'origine de sa maladie renvoie à une forme de «difficile à dire » (Gardin, 2006), qu'il s'agisse du contenu de la recherche causale ou de la gestion de l'interaction avec l'interviewer médecin.

Les différentes voix convoquées par F. correspondent en effet à des places discursives qui peuvent être vues simultanément comme des places sociales. La voix de la connaissance sur soi par familiarité, originale en ce qu'elle apparaît comme la seule voix qui permet de parler de soi sans emprunter aux autres, acquiert ici une forme de légitimité en étant mise en regard celle des autres, et en particulier celle qu'elle attribue à son interviewer. L'introduction réitérée de cette voix très personnelle (assumée au niveau énonciatif de façon faible mais cependant nettement actualisée) pour «concurrencer» la voix médicale modifie en effet fortement l'asymétrie sociale attendue dans une interaction entre médecin et non-médecin : l'orchestration des voix par F. a finalement une fonction d'argumentation et de mise en scène positive de soi. Cette orchestration joue clairement un rôle de repositionnement social dans l'espace de suspension ponctuel permis dans et par l'entretien.

\section{Références bibliographiques}

Anscombre, J.-C. (1995). Théorie des topoï, Paris : Kimé.

Authier-Revuz, J. (1982). Hétérogénéité montrée et hétérogénéité constitutive; éléments pour une approche de l'autre en discours, DRLAV, 26, 91-151.

Authier-Revuz, J. (1984). Hétérogénéité(s) énonciative(s). Langages, 73, 98-111.

Authier-Revuz, J. (1995). Ces mots qui ne vont pas de soi. Paris : Larousse.

Bakhtine, M. (1984 [1979]). Esthétique de la création verbale. Paris : Gallimard.

Bakhtine, M. (1970 [1963]). La poétique de Dostö̈evski. Paris : Seuil.

Bakhtine, M. (1977 [1929]). Le Marxisme et la philosophie du langage. Essai d'application de la méthode sociologique en linguistique. Paris : Minuit.

Bailly, D., Regnault, N., Danel T. \& Parquet P. J. (1991). Troubles du comportement alimentaire :des liens étroits avec la toxicomanie et certaines formes d'alcoolisme. Annales de Psychiatrie, 6, (1), 41-50.

Bally, C. (1965 [1934]), Linguistique générale et linguistique française. Berne : Francke.

Benveniste, E. (1966). Problèmes de linguistique générale. Paris : Gallimard.

Bres, J. (1998). Entendre des voix : de quelques marqueurs dialogiques en français. In J. Bres, R. Delamotte-Legrand, F. Madray-Lesigne, P. Siblot, L'autre en discours, Montpellier-Praxiling et Rouen-Dyalang, p. 191-212.

Bres, J. (2005). Savoir de quoi on parle : dialogue, dialogal, dialogique ; dialogisme, polyphonie. In J. Bres, P. P. Haillet, S. Mellet, H. Nølke, \& L. Rosier (Eds.), Dialogisme et polyphonie. Approches linguistiques. Bruxelles : De Boek Duculot, p. 47-61.

Bres, J. \& Nowakowska, A. (2006). Dialogisme : du principe à la matérialité discurssive. Recherches linguistiques, $n^{\circ}$ $28,143-164$.

Bronckart, J.-P. (2004). Agir et discours en situation de travail. Cahiers de la section des sciences de l'éducation, 103, 147-184. 
Carcassonne, M. (1996). Parler de soi quand on est dépendant : analyse interactive et énonciative. Langage et Société, $76,5-41$.

Carcassonne, M. (1997). Énonciation, mouvements et temporalités dans des récits de vie recueillis en interaction, Thèse de doctorat de linguistique, Université René Descartes-Paris V.

Carcassonne, M. (2004). De la dimension temporelle des affects vécus à celle des affects racontés : quelles médiations? In R. Delamotte-Legrand G. (Ed.), Les médiations langagières, vol. 1, Des faits de langue aux discours, Actes du colloque international La médiation: marquages en langue et en discours. Dyalang CNRS: Publications de l'Université de Rouen, 403-411.

http://basepub.dauphine.fr/bitstream/handle/123456789/1234/affects $\% 20 \mathrm{dits} \% 20$ (Rouen) $\% 20$ pagin $\% \mathrm{C} 3 \% 83 \% \mathrm{C}$ $2 \%$ A9.pdf? sequence $=1$

Carcassonne, M. (2006). Présentation de soi dans l'entretien clinique : narration, explication, interprétation. In M. Grossen, A. Salazar-Orvig (dirs.), L'entretien clinique en question: analyse des interactions d'un genre communicatif hétérogène. Belin (coll. Belin Sup), 213-231.

http://basepub.dauphine.fr/bitstream/handle/123456789/1324/belinPDF-Pagination.pdf?sequence=1

Carcassonne, M. (2007). Entretiens, émotions et récit de soi. In Vox Poetica, Passion et narration, Revue en lignes, http://www.vox-poetica.org/t/pas/index.html

Carcassonne, M. (2008). Entretiens de recherche en psychopathologie : une approche par les mouvements de genres. Langage \& Société, 123, 87-103.

Carcassonne, M. \& Servel, L. (2009). Dispositif d'enquête et production d'acteurs sociaux : apport d'une analyse socio-langagière. Activités, 6 (2), 44-56. http://www.activites.org/v6n2/v6n2.pdf

Carcassonne, M. \& Valleur M., (1997). Discours et conduites addictives. In A. Blanchet (dir.), Champs de recherche en psychologie clinique. Paris : Dunod, p. 69-95.

Carcassonne, M., Salazar-Orvig, A. \& Bensalah, A. (2001). Des récits dans des entretiens de recherche : entre narration et interprétation. Revue québécoise de linguistique, vol.29, $n^{\circ}$ 1, 97-122.

Charles-Nicolas, A. \& Valleur, M. (1982). Les conduites ordaliques. In C. Olievenstein, La vie du toxicomane, Séminaire de l'hôpital Marmottan. Paris : PUF, 82-99.

Cicourel, A. (2002). Le raisonnement médical. Paris : Seuil.

Clavreul, J. (1978). L'ordre médical. Paris : Seuil.

Clot, Y. (1999). La fonction psychologique du travail. Paris : PUF.

Cole, M. (1994). A conception of culture for a communication theory of mind. In D. R. Vocate (ed.), Interpersonal Communication. Different voices, different minds. Erlbaum : Hillsdale.

Cole, M. (1995). Cultural-historical psychology : a meso-genetic approach. In L.M.W. Martin, K. Nelson \& E. Tobach (Eds), Sociocultural psychology. Theory and practice of doing and knowing. Cambridge, Cambride University Press, 168-204.

Coste, D., Mochet, M. A., Papo, E. (1986). Variations sur entretiens. Etudes de linguistiques appliquées, 63, 72-82.

Danon-Boileau, L. (1982). Produire le fictif. Paris : Klincksieck.

Danon-Boileau, L. (1987). Enonciation et référence. Paris-Gap : Ophrys.

Drew, P. (1991). Asymmetries of knowledge in conversational interactions. In I. Markovà I, K. Foppa (eds), Asymmetries in Dialogue. Harvester Wheatsheaf, Herfordshire (Great-Britain), 38-48.

Eco, U. (1992 [1990]). Les limites de l'interprétation. Paris : Grasset.

Erhenberg, A. (1995). L'individu incertain. Paris : Hachette.

Flahaut, F. (1978). La parole intermédiaire. Paris : Seuil.

François, F. (1984). Interprétation linguistique et psychopathologie. L'Evolution psychiatrique, tome 49, fascicule 2 , $15-450$. 
François, F. (1989). De quelques aspects du dialogue psychiatre-patient. Places, genres, mondes et compagnie. C.A.L.A.P., $n^{\circ} 5,39-89$.

François, F. (1993). Pratiques de l'oral. Paris : Nathan.

François, F. (1994). Morale et mise en mots. Paris : L'Harmattan.

François, F. (1995). Quelques remarques sur la notion d'interprétation ou du dialogue quotidien comme modèle d'interprétation. Cahiers de l'ILSL, $n^{\circ} 6,97-129$.

François, F. (2009). Essais sur quelques figures de l'orientation; hétérogénéités, mouvements et styles. Paris : Lambert-Lucas.

Gadamer, H. G. (1960). La compréhension dans les sciences humaines. In Vérité et méthode. Paris : Seuil, 103-226.

Gardin, B. (1978). Volochinov ou Bakhtine? La pensée, 197. Repris in B. Gardin (textes rassemblés par Nanon Gardin et Frédéric François), 2006, Langage et luttes sociales. Limoges : Lambert Lucas, 69-86.

Gardin, B. (1988). Le dire difficile et le devoir dire. DRLAV, 39. Repris in B. Gardin (textes rassemblés par Nanon Gardin et Frédéric François), 2006, Langage et luttes sociales. Limoges : Lambert Lucas, 133-148.

Goodman, A., (1990). Addiction : definition and implications. British Journal of Addiction, 85, 1403-1408.

Grunig, B.N. et Grunig, R. (1985). La fuite du sens, La construction du sens dans l'interlocution. Paris : Hatier.

Halbwachs, M. (1950). La mémoire collective, Paris, PUF.

Ivanova, I. (2003). Le dialogue dans la linguistique soviétique des années 1920-1930. Cahiers de l'Institut de linguistique et des sciences du langage (Université de Lausanne), 14, 157-182.

Jauss, H. R. (1978). Pour une esthétique de la réception, Paris : Gallimard.

Kerbrat-Orecchioni, C. (1980). L'énonciation. De la subjectivité dans le langage. Paris, A. Colin.

Kerbrat-Orecchioni, C. (1989). Théorie des faces et analyse conversationnelle. In I. Joseph (ed.), Le parler frais d'Erving Goffman. Paris : Minuit, 155-179.

Kerbrat-Orecchioni, C. (1990). Les interactions verbales, T.1, 2 Paris : Armand Colin.

Kuyumcuyam, A. (2006). Les narrations comme mentions. Polyphonies et narrations. Recherches linguistiques, $n^{\circ}$ $28,143-164$.

Mc Dougal, J., Marinov V., Brelet-Foulard F., (2001). Anorexie, addictions et fragilité narcissique. Paris : PUF.

Markovà, I. (2007 [2003]). Dialogicité et représentations sociales. Paris : PUF.

Markovà, I. \& Foppa, K. (eds). (1991). Asymmetries in dialogue. New-York : Harvester.

Martin, R. 1983, Pour une logique du sens. Paris : PUF.

Mochet, M.-A. (1989). Aise/Malaise en situation d'entretien. BUSCILA, L'interaction, 178-195.

Mondada, L. (1989). Autour de la construction dialogique du sens. Actes du Troisième Colloque Régional de Linguistique, Strasbourg: Université des Sciences Humaines et Université Louis Pasteur, 46-57.

Mondada, L. (2001). Pour une linguistique interactionnelle. Marges Linguistiques $n^{\circ} 1$, http://www.margeslinguistiques.com

Olivenstein, C. (dir.) (1982), La vie du toxicomane. Paris : PUF.

Ricœur, P. (1990). Soi-même comme un autre. Paris : Le Seuil.

Rogoff, B. (1990). Apprenticeship in thinking. Cognitive development in social context. New-York: Oxford University Press.

Sacks, H., Sschegloff E., Jefferson G. (1974). A simplest systematics for the organization of tum-taking in conversation. Language, 50, 696-735.

Salazar-Orvig, A. (1999). Les mouvements du discours. Style référence et dialogue dans des entretiens cliniques. Paris : L'Harmattan. 
Salazar-Orvig, A. (2003). Le devenir de la parole de l'autre : pour une typologie des mouvements interprétatifs dans l'entretien clinique. Psychologie de l'interaction, 15-16, 195 - 221

Salazar-Orvig, A. (2008). La notion de déplacement dans une approche dialogique du dialogue. In R. DelamotteLegrand, A. Salazar-Orvig et C. Hudelot (eds) (2008), Dialogues, mouvements discursifs, significations. CortilWodon : E.M.E., 15-30.

Salazar-Orvig, A. \& Grossen, M. (2008). Le dialogisme de l'entretien clinique. Langage et Société, 123, 37-52.

Sarfati, G. E. (1997). Eléments d'analyse de discours. Paris, Nathan.

Schneuwly, B. (1994). Genres et types de discours. In Y. Reuter (éd), Les interactions lecture-écriture. Berne : Peter Lang, 155-174.

Thomassin, P. (1999). Déconstruction et reconstruction de l'identité narrative au cours d'une psychothérapie. Psychologie française, 44 (4), 371-381.

Valleur, M. (1994). Toxicomanie et mort, addictions et conduites de risque. Rapport de Recherche pour le Ministère de l'Enseignement Supérieur et de la Recherche. GRECO. Paris. http://www.hopitalmarmottan.fr/spip/IMG/pdf/greco1.pdf

Valleur, M. (1997). Discours et conduites addictives. In A. Blanchet (Ed.), Champs de recherche en psychologie clinique, Paris : Dunod, 69-95.

Vincent, D. (2006). Polyphonie et interaction. Recherches linguistiques, $n^{\circ} 28,127-142$.

Vion, R. (1999). Pour une approche relationnelle des interactions verbales et des discours. Langage et Société, 86, 95129.

Vion, R. (2001). Modalités, modalisations et activités langagières. Marges Linguistiques, 2, 209-231, http://www.revue-texto.net/marges/marges/Documents\%20Site\%200/00_ml112001/00_ml112001.pdf

Vion, R., Rouveyrol, L., Maury-Rouen C., Bremond C., Boström E., Assef C., Noël-Jorand M.C. (2001). Outils linguistiques pour l'analyse linguistique des discours et des émotions. Revue française de psychiatrie et de psychologie médicale, 5 (49), 49-56.

Vygotski, L. S. (1985 [1934]). Pensée et Langage. Paris : Éditions sociales.

Weinrich, H. (1989). Grammaire textuelle du français. Paris : Didier.

Wertsch, J. V. (1991). Voices of the mind: a sociocultural approach to mediated action. Cambridge : Harvard University Press.

Zafiropoulos, M. (1988). Le toxicomane n'existe pas. Paris : Navarin.

${ }^{1}$ Comme nous l'avons déjà noté ailleurs (Carcassonne \& Servel, 2009, p. 46), plusieurs auteurs (par ex. Gardin, 1978 ; Bronckart, 2004 ; Ivanova, 2003) ont analysé le fait qu'un certain nombre d'ouvrages publiés sous le nom de Bakhtine ont en fait été écrits par Voloshinov. Pour la commodité du renvoi aux ouvrages, publiés uniquement sous le nom de Bakhtine, nous utiliserons ce seul nom.

${ }^{2}$ Dans cet article, l'hétérogénéité constitutive du discours est appréhendée en croisant les théories du dialogisme (Bakhtine) et de la psychanalyse (à partir d'une lecture de Freud marquée par celle de Lacan). Pour Authier-Revuz, la prise en compte de cette hétérogénéité constitutive se situe à l'extérieur du linguistique mais constitue un ancrage nécessaire pour la description linguistique des formes d'hétérogénéité montrée.

${ }^{3}$ La notion de «résidu» est de ce point de vue une reprise-modification de l'argument et alia de la rhétorique. Comme le souligne François (1994 : 64), « on voit mal ce que serait un discours qui n'aurait rien oublié ».

${ }^{4}$ Dans la terminologie du Cercle de Bakhtine, lorsque les voix apparaissent sur un pied d'égalité et ne sont pas hiérarchisables (comme Bakhtine (1970 [1963]) l'a mis en évidence à propos des romans de Dostoïevski), elles sont alors rapportées à une forme particulière de dialogisme, dénommée « polyphonie ». Soulignons cependant qu'il est difficile d'envisager une polyphonie des voix absolue, sans aucune domination d'aucune sorte : par exemple n'y aurait-il pas (relative) domination de celui qui est le premier à aborder un thème ? Ou celle, contradictoires, de celui qui pose les questions ou de celui qui est capable d'apporter les réponses? (Sur l'analyse de la polyphonie dans l'interaction, cf. Vincent D., 2006). 


\footnotetext{
${ }^{5}$ Vygotski a insisté sur l'importance de l'interaction avec autrui dans le développement cognitif de l'individu : sa perspective entre en résonnance celle de Bakhtine, et cela alors même que les deux auteurs, pourtant contemporains, ne se sont pas connus.

${ }^{6}$ La notion de «genre » est ici prise au sens de Bakhtine-Voloshinov qui a décrit comment chaque « sphère » de l'activité humaine « (...) comporte un répertoire de genres de discours qui va se différenciant et s'amplifiant au fur et à mesure que se développe et se complexifie la sphère donnée » (Bakhtine 1984, p. 265) : les « genres » de discours correspondent à des types relativement stables d'énoncés en tant qu'ils sont liés à une pratique sociale et/ou une activité. Ils sont issus de modèles historiquement constitués qui « orientent 》 (Schneuwly, 1994: 160) nos processus de réception et surtout de production des discours. Sinon, "l'échange verbal serait quasiment impossible » (Bakhtine, $1984: 285)$.
}

${ }^{7}$ L'expression « mise en mots» pointe l'activité de «choix» des mots et de leurs agencements, qui peut être différent d'un locuteur à un autre et d'un moment à un autre pour un même locuteur. La "mise en mots » ne se conçoit pas comme un « calque » du réel mais comme un processus comportant une part de créativité ou du moins de spécificité de la façon de dire d'un locuteur. En ce sens, la notion de mise en mots est liée à celle de « style discursif» (François, 1994).

${ }^{8}$ Flahaut estime que c'est au niveau de cette articulation intermédiaire que s'établit l'identité de l'individu.

${ }^{9}$ "Il existe, dans l'abstrait, la position du troisième qu'on identifie à la "position objective " en tant que telle, à la " connaissance scientifique ». La position du troisième est entièrement justifiée là où un individu peut se mettre à la place d'un autre individu, là où les individus sont absolument interchangeables, ce qui est possible et fondé seulement dans le cas où l'on cherche une solution à des problèmes qui ne sollicitent pas la personne dans sa totalité et sa non-reproductibilité, autrement dit, là où l'homme se spécialise, exprimant seulement une partie détachée de son tout, de sa personne, là où sa qualité $d$ ' "ingénieur», de "physicien», etc., sera substituée à son moi » (Bakhtine 1984 : 364).

${ }^{10}$ En s'inspirant de son analyse, nous pouvons constater la différence entre (1) «Il croque une pomme à pleines dents » et (2) «Il croque la vie à pleines dents»: (1) implique une référence hic et nunc " pomme » interprétant (déteignant sur) "croque» comme une action ponctuelle;(2) implique une référence générique, "la vie» interprétant « croquer » comme une action durative. " Pomme » et " vie » font donc changer le sens de « croquer ».

${ }^{11}$ François retient les affinités mise en évidence par Danon-Boileau en les intégrant dans une perspective textuelle telle que la définissait Weinrich : "on parlera d'affinités pour désigner des éléments de sens non localisables, mais qui courent dans le texte. On préfère ce terme à "isotopie » car il implique un air de ressemblance, non un trait réellement commun. Ainsi, il y a affinité lexico-grammaticale entre l'imparfait et le verbe dans "je me promenais tranquillement », anti-affinité ou moins d'affinité dans "je me promenai tranquillement " (...). De même peut-on parler d'affinité textuelle pour référer au fait que « ceci étant dit », on attend, sans que ce soit nécessaire, plutôt telle suite que telle autre» (1994: 42).

${ }^{12}$ Jauss (1978 : 284) était dans une perspective d'interprétation des textes littéraires qui peut être transposée, comme pour Bakhtine, à celle des discours produits en interaction. Il a formulé l'idée selon laquelle on ne peut comprendre un texte que si l'on a compris à quelle question il répond; et l'on ne peut reconstituer cette question qu'en opérant une fusion synchronique de deux horizons d'attente : l'un antérieur au texte et l'autre que le texte fait apparaître. Il s'est inspiré de Gadamer (1960) qui proposait une fusion diachronique des horizons.

${ }^{13}$ La recherche du GRECO s'inscrivait à l'époque sur un arrière-fond de controverse entre pouvoirs publics et corps médical (et même au sein du corps médical), sur les modalités de prise en charge des addictions. Pour les uns, l'addiction est une maladie, voire un délit, à éradiquer de façon indifférenciée, en prescrivant un produit de substitution pour induire un changement de comportement du « délinquant» (cf. par ex. Hefez, Libération du 25 juillet 1994); pour les autres, elle est causée par la rencontre entre une personnalité, un produit et un contexte social (Olivenstein 1982, Zafiropoulos 1988), et donc en partie due à un malaise généré par la société elle-même (Erhenberg, 1995). Pour ce dernier, un comportement addictif est à voir comme le propre d'un individu « souffrant» s'opposant à une individu "conquérant", les deux types d'individus correspondant à deux facettes d'un «gouvernement de soi » autrefois pris en charge par la société, alors qu'aujourd'hui chacun doit désormais se construire une place « incertaine » $(1995: 18)$.

${ }^{14}$ In Valleur, $1994: 72-74$ et 168-169. 
${ }^{15}$ Carcassonne, 1996, 1997, 2004, 2006, 2007, 2008, Carcassonne \& Valleur, 1997 ; Carcassonne, Salazar-Orvig \& Bensalah, 2001.

${ }^{16}$ Les numéros correspondent à la numérotation des lignes dans l'ordre de leur apparition au cours de la transcription. Les retours à la ligne constituent une interprétation relativement subjective des courbes intonatives qui n'ont pas été transcrites). Une transcription proche du français écrit a été adoptée ici pour des raisons liées au mode d'enregistrement; les aspects intonatifs et corporels n'ont pas pu être pris en compte ici, même s'ils nous pour nous d'une grande importance pour l'interprétation et qu'il y a dans ce type d'entretien un dialogisme important aux niveaux de l'intonation, des regards, de l'attitude de corporelle en général. Les questions de l'interviewer apparaissent en gras.

${ }^{17}$ Cet exemple n'est toutefois peut-être pas aussi généralisable aujourd'hui que dans les années 80, la doxa sur ce thème ayant évolué ces dernières années. 